\title{
Reading and cognitive capacity usage: Adjunct question effects
}

\author{
BRUCE K. BRITTON, ALAN PIHA, JOHNNY DAVIS, and ELLEN WEHAUSEN \\ University of Georgia, Athens, Georgia 30602
}

\begin{abstract}
Cognitive capacity usage during reading was measured in two experiments. During reading of a 27-page passage on marine biology, text-relevant inserted questions, irrelevant questions, or no questions were answered by undergraduates. Cognitive capacity usage increased when text-relevant questions began but not when irrelevant questions began or when no questions were given. The increase in cognitive capacity usage was largest on pages immediately following questions. Cognitive capacity is used in the elaborative stages of text processing.
\end{abstract}

Reading requires cognitive capacity. With increases in the amount of cognitive capacity devoted to reading, less is available for reacting to other events in the environment. A reader at the "good part" of a novel is likely to react very slowly to external stimuli that would normally call forth a quick reaction. Perhaps it is possible to measure how much cognitive capacity readers are using by measuring how quickly they react to other stimulation. The more cognitive capacity they are using for processing the reading material, the slower will be their reaction to another stimulus. This measurement procedure for quantifying the usage of cognitive capacity has been applied to measure the cognitive capacity demands of a wide variety of tasks other than reading (reviewed by Kahnemann, 1973; Kerr, 1973); in the experiments reported here, it was applied to reading. There was a primary task, reading, and a secondary task, reacting to occasional clicks by pressing a button. The tasks were concurrent. The basic assumption is that the more cognitive capacity the reader is using for reading, the less spare cognitive capacity is left over for reacting to the clicks, and consequently, the slower the reaction time. The rationale for this involves several assumptions: that the central cognitive processor has limited capacity, that the capacity is fixed, that the tasks of reading and of responding to clicks both require space in the same central processor, that as more capacity is devoted to reading less is available for reacting to the clicks, and that reaction time to the click task reflects the mental capacity devoted to it.

At the anecdotal level, the amount of cognitive capacity devoted to a text by a reader appears to play an important role in determining how much is learned from the text. At one extreme, most people

This research was supported by a grant from the University of Georgia. Appreciation is due to Ben Stephens and Tim Casey for assistance in testing subjects, to Ellen Gagné, Milton Hodge, and reviewers for helpful comments, and to Robert Westbrook and Robbie Ann Ziegler for assistance in data analysis. Ms. Ziegler was supported by National Science Foundation Grant SMI 77-00233. have experienced reading episodes where the reader's mind wanders elsewhere while the lines of text march before the eyes. Little or no cognitive capacity is devoted to the text and little or nothing is learned. At the other extreme, readers deeply absorbed in a text tend to learn from it.

To test the hypothesis that the amount of cognitive capacity devoted to reading is related to the amount learned from it, a simple situation was chosen in which it was clearly and firmly established that the amount learned from a text was markedly affected by a straightforward manipulation of the learning situation. The learning manipulation was the adjunct question procedure developed by Rothkopf (1966). Difficult questions about detailed information in the text (names, dates, facts) are asked after the portion of the text they refer to. The subjects must answer the questions without looking back at the text, and then they read on. When the next part of the text is read, subjects learn much more from it. Anderson and Biddle's (1975) review of this literature led to the firm conclusion that inserting questions in the text after the relevant parts of the text and requiring subjects to answer the questions without looking back is an effective procedure for facilitating learning of later parts of the text.

These experiments were concerned with the effects of adjunct question treatments on cognitive capacity usage. Comparisons were made between the cognitive capacity devoted to reading by groups that periodically answered adjunct questions relevant to text that had been read just previously, and by control groups that read the text without questions or with irrelevant questions. It was expected that, just as learning in groups with adjunct questions exceeds learning in reading-only control groups (Anderson \& Biddle, 1975), more cognitive capacity would be used for reading in the questioned groups than in the control groups.

\section{EXPERIMENT 1}

\footnotetext{
Method

Subjects. The subjects were 48 undergraduates participating as part of a research requirement for introductory courses.
} 
Subjects were assigned to conditions in the order of their appearance in the laboratory, with the restriction that the nth subject was not assigned to a group until the $n-1$ th subject had been assigned to the other group.

Conditions. There were two conditions. In both conditions the first 15 pages of text were read without questions. The no question group then continued to read the remaining 12 pages without questions, while the question group was required to answer six short answer questions after every 3 of the remaining 12 pages of text.

Materials. A 27-page passage on marine biology from The Sea Around Us (Carson, 1951) was used. It was the same section used by Rothkopf (1966). Rothkopf supplied the short answer questions used in the present study. This particular passage and short answer questions on it have also been used in a study of the effects of inserted questions on learning by McGaw and Grotelueschen (1973). A typical paragraph and short answer questions illustrate the nature of the task.

"The last traces of plant life are left behind in the thin upper layer of water, for no plant can live below about 600 feet even in very clear water, and few find enough sunlight for their food manufacturing activities below the first 300 feet called the photic zone. Only $2 \%$ of the entire ocean bottom is within the photic zone. Since no animal can make its own food, the creatures of the deeper waters live a strange, almost parasitic existence of utter dependence on the upper layers" (Carson, 1951).

"In the sea, no plant life is found below feet. The first 300 feet in the sea is called the zone. What percentage of the ocean bottom has enough sunlight so that food can be manufactured by most organisms? The source of food for animals in the deep parts of the sea is

The materials were arranged in booklets. For all groups the first 18 pages of the booklets contained no questions. They consisted of a cover page, the first 3 pages of text, a page asking the subject to wait briefly, the next 12 pages of text, and a page asking the subject to wait briefly. After this point the booklets differed. For the question group, there were six questions on the immediately preceding three pages of text, then three pages of text, then six questions on those three pages, and so on for the remaining pages of text. Subjects were not permitted to look back at the preceding text while answering the questions. The questions were those provided by Rothkopf (1966), with additional ones constructed by the first author, to make up six for each segment. Of the 30 questions, 13 had answers requiring technical terms or proper names, 9 required numbers as answers, and 8 could be answered with common words. For the no question group, the booklets were composed of the remaining 12 pages of text.

Apparatus. The click was made by the hand switch used to turn on a clock reading in hundredths of a second. The subject had been instructed to keep a telegraph key depressed and to let it up when the click was heard. Then the experimenter reset the hand switch and clock and said, "Okay." This was the subject's signal to depress the key again. This seemed to be a natural and compatible procedure, and once it had been practiced a few times, there was no difficulty in doing it while reading. A tape recorder played white noise at a low level throughout the session to mask ambient noise.

Procedure. At the beginning of the experiment, each subject was asked to read the material so that he or she would be prepared to take a test over it at the end of the experiment. After the first three pages had been read, the experimenter asked the subject to wait briefly while he completed the record of the first part of the experiment. Then the subject was informed about the click probes, instructed in the use of the reaction time key, and given practice in responding to the clicks. Then the next 12 pages were read. All subjects were then asked to stop. In the no question group, subjects were told that the next part of the experiment would continue in the same way as the preceding part, and they proceeded to read the next 12 pages as before.

In the question after group, subjects were informed that in the next part of the experiment, they would receive six short answer questions every few pages and that they would not be permitted to refer to the text. Then they continued. The first six questions occurred immediately. Subjects answered the questions orally and were told whether the answer was correct or incorrect. If incorrect, a correct answer was provided.

After all text and questions had been completed, all subjects were asked to listen for the click without reading for three trials. Reaction time to the clicks was recorded.

Click probe placement. On each of pages 4-27, each subject who performed the secondary task was programmed to receive one, two, or three click probes. On each set of three pages, (ne page had one probe, one page had two, and one page had $t$. ree. The pattern of probes was predetermined according to four schedules. For each subject the schedule was chosen randomly, with the restriction that $\mathbf{n}-1$ subjects in each group had been exposed to each schedule before the nth subject was exposed to a schedule. For each subject, the probe pattern for the first 12 pages of probed text was identical to the probe pattern for the last 12 pages of probed text.

Pilot work showed that there was wide variability in reading speed in this population. If the click probes had been inserted on a schedule based on the time elapsed since the last probe, fast readers would have had many fewer probes than slow readers. To avoid this, probes were arranged so that each subject received approximately the same number of probes.

Average reading speed was determined on the first three pages of text, during which no probes were presented. Then the average speed was reduced by $20 \%$ and divided into thirds. When there were three probes on a page, they were at each third. Thus, each subject received about one-third of the probes one-third of the way through the page, one-third two-thirds of the way through the page, and one-third near the end of the page. This procedure was successful in approximately equalizing the number of probes each subject received. The average subject received a probe every $34 \mathrm{sec}$.

\section{Results and Discussion}

Figure 1 shows the average reaction time for each page for the two groups. Vertical lines indicate occasions when questions were inserted in the question condition. Over the first 12 pages of probed text, when no questions were present for either group, the groups did not differ, and there were no substantial differences between pages. These impressions were confirmed by the results of a two-factor analysis of variance, with groups as a between-subjects factor and pages as a within-subjects factor. There was no effect for groups or for pages or the interaction.

Over the second 12 pages of text, the question group took considerably more time to react to the clicks (mean $=.46 \mathrm{sec}$ ) than the no question group (mean $=.41 \mathrm{sec})$. This difference was reliable, as shown by the results of a Groups by Pages analysis in which there was a significant effect for groups $[F(1,46)=4.18$, $\mathrm{MSe}=96.36]$. (All effects were significant at $\mathrm{p}<.05$.) This analysis also revealed a reliable effect of pages $[\mathrm{F}(11,506)=3.67, \mathrm{MSe}=65.76]$ and no interaction. The source of the reliable effect of pages has not been determined.

It is conceivable that the effect of questions on reaction time could be due, not to the questions 


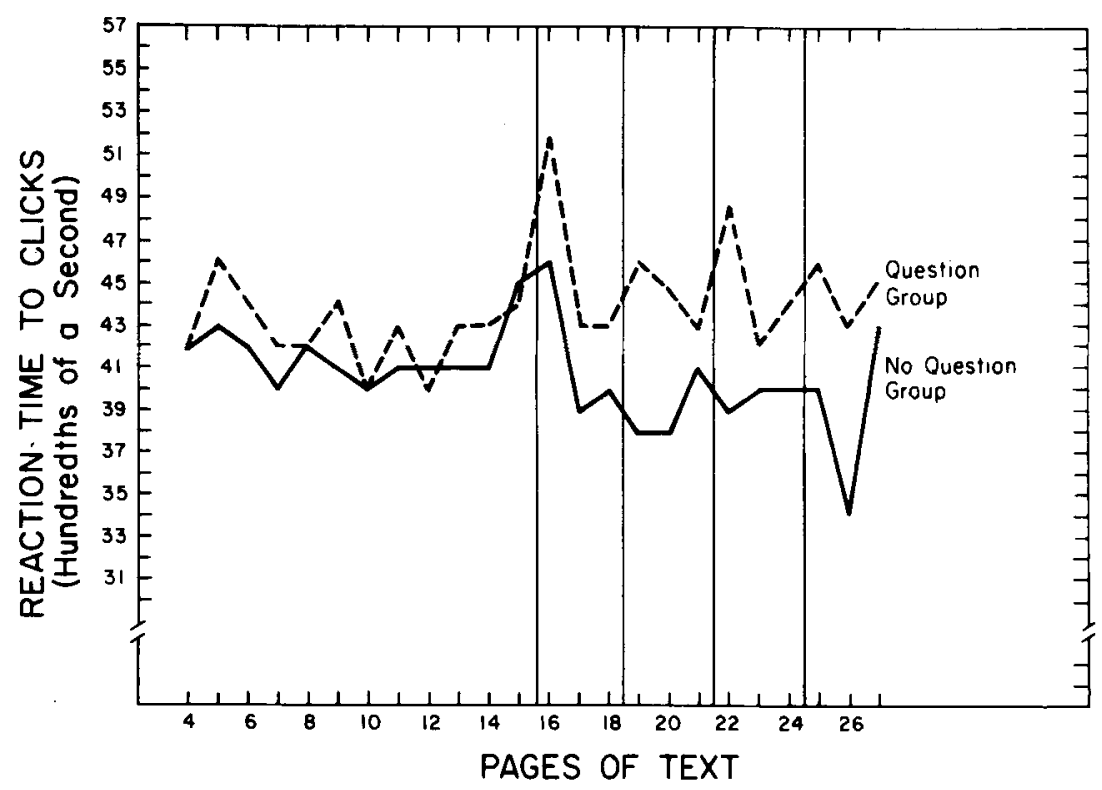

Figure 1. Experiment 1 reaction time to click probes in questioned and unquestioned groups. Questions occurred at vertical lines.

themselves, but to the interruptions of the reading task that occurred concurrently. This hypothesis can be tested by considering the case in which the no question group was interrupted. This occurred after the 12th page of probed text. If the interruption alone was responsible for the increase in reaction time for the question group, then reaction time in the no question group should increase from the page before to the page after the interruption. The increase was slight in absolute magnitude and was not reliable. However, it should be noted that, in contrast to the experimental group, the interruption for the control group was comparatively brief and une ventful and was not repeated periodically. These features of the interruption may be important. In Experiment 2, this issue is examined more thoroughly.

Trends in reaction time over the 1-h session were examined. In the question group, reaction time increased when questioning began. Tinis increase was reliable, as shown by a $t$ test comparing the average reaction time in the first half of the experimental session $($ mean $=43$ ) with that in the second half (mean $=46$ ) $\left[\mathrm{t}(23)=2.07, \sigma_{\text {est }}=1.41\right]$. A similar test in the no question group showed that the slight decline (Part 1, mean $=42 ;$ Part 2, mean $=41$ ) was not reliable.

At the end of the experiment, reaction times without reading were taken for both groups. For the no question group, the average was $.33 \mathrm{sec}$. For the question group, the average was $.34 \mathrm{sec}$. These were not reliably different. These figures may be compared with the best estimate of secondary task reaction time while reading, which is the overall average reaction time during reading of the no question group, $.41 \mathrm{sec}$.

Reading time is generally longer in adjunct question groups than in reading-only control groups (Anderson \& Biddle, 1975; Carver, 1972; cf. Rothkopf, 1974), and this was also found in the present experiment. The experimenter recorded the number of seconds taken to read each page (question-answering time was not included).

The average reading time per page during the second part of the experiment in the question group (mean $=85 \mathrm{sec}$ ) exceeded that in the no question group (mean $=73 \mathrm{sec}$ ). The difference was significant, but barely so, at the .05 level $[\mathrm{t}(46)=1.99$ (one-tailed test)]. Reading rates for the question group (200 words/min) and the no question group (232 words/ $\mathrm{min}$ ) were consistent with average college student reading rates (Coke, 1974), indicating that the secondary task did not markedly alter normal reading processing as reflected by reading time.

Adjunct questions caused increases in reaction time to the secondary task. These increases presumably reflected an increase in the amount of cognitive capacity used for reading. However, there are alternative explanations for this increase. The second experiment included additional control groups to investigate these alternatives.

\section{EXPERIMENT 2}

In Experiment 1, reaction time to the click probes increased when questions began. This increase could have been caused by either of two factors. Two things happen when questions begin: (1) Students start to study harder (Anderson \& Biddle, 1975) and (2) the reading task is interrupted by the questions. The increases in reaction time during reading could have been 
caused either by (1) studying harder because of the questions, or (2) having to pick up the thread of the narrative because of the interruptions, or by both factors. In Experiment 2, a condition was included in which the reading task was interrupted by questions, but the questions were not relevant to the text (e.g., name three political figures). In this condition there were interruptions, but nothing to cause the subjects to study harder. Cognitive capacity usage in this condition was compared with that in a group that received text-relevant questions (question after group) and no questions (no question with probes group).

Experiment 2 also investigated the issue of whether the secondary task itself influenced the normal cognitive processing involved in reading. An additional control condition was included in which there was no secondary task (the no question without probes group). This group was compared with the no question with probes group. The only difference between these groups was that one included the secondary task while the other did not. If the secondary task influenced normal reading processing, differences between the two groups on some aspect of performance should appear. For both groups, measurements were taken of reading time and score on a final eight-question short answer test on the passage.

\section{Method}

Subjects. The subjects were 72 undergraduates participating as part of a research requirement for introductory courses. There were 18 subjects in each group. Subjects were assigned to conditions in the order of their appearance in the laboratory, with the restriction that the nth subject was not assigned to a condition until the $n-1$ th subject had been assigned to the other condition.

Conditions. There were four conditions. The question after condition was identical to the question condition of Experiment $i$. The no question with probes condition was identical to the no question condition of Experiment 1 . The irrelevant question condition was the same as the question after condition, except that the questions tested general knowledge that was not relevant to the topic of the text (e.g., name two American railroads, or popular songs, or best-selling books, etc.). The no question without probes condition was identical to the no question with probes condition except that no click probes were administered. For this condition, the section of the instructions about probes was omitted.

Experiment 2 was identical to Experiment 1 in materials, apparatus, procedure, and click probe placement.

\section{Results and Discussion}

Figure 2 and Table 1 show the basic data. Reaction

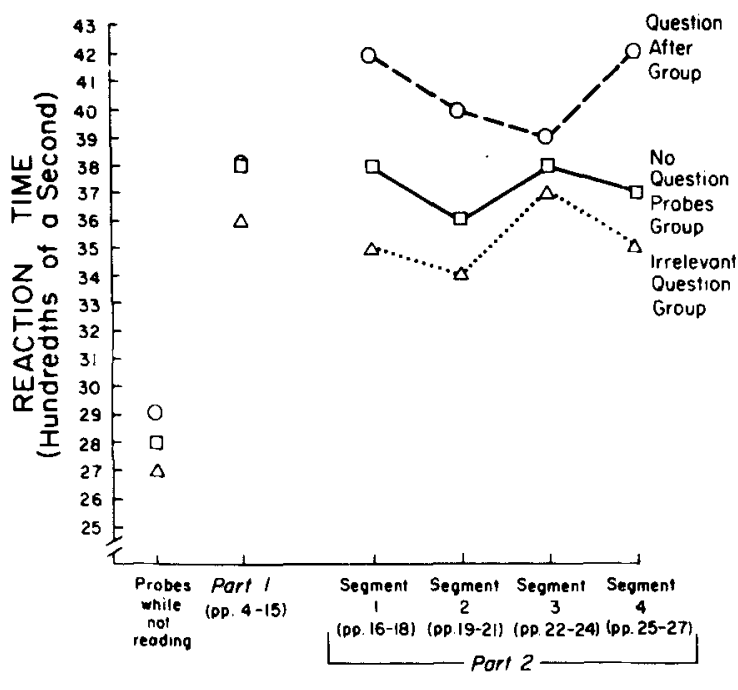

PART OF EXPERIMENT

Figure 2. Experiment 2 reaction time to click probes in questioned and unquestioned groups.

times taken while subjects were not reading did not differ reliably, and the average reaction times to probes did not differ over pages $4-15$, during which the three probed groups were treated identically; these data indicate the groups were equivalent.

Over the second 12 pages of probed text, the question after condition reacted more slowly to probes than the no question with probes condition, as shown in a conditions by segments analysis of variance of those groups: There was a reliable effect of conditions $[\mathrm{F}(1,3)=5.84, \quad \mathrm{MSe}=76.73$ (all results $\mathrm{p}<.05)]$ and there were no effects of segments or the interaction.

One hypothesis is that the increases in cognitive load in the question after condition were due to the interruptions of the reading task which occurred concurrently with the questions. This was tested by comparing the irrelevant question condition with the no question with probes condition. The hypothesis implies that the irrelevant question condition should have longer reaction times than the control group; in fact these groups did not differ, as shown by a conditions by segments analysis, which showed no reliable effects for conditions, segments, or the interaction.

Examination of trends over the entire experimental session revealed that reaction times to probes increased when questions began to occur in the question after

Table 1

Average Reaction Time to Click Probes, Reading Time, and Test Scores for Experiment 2

\begin{tabular}{|c|c|c|c|c|c|}
\hline \multirow[b]{2}{*}{ Condition } & \multicolumn{2}{|c|}{ Average Reaction Time } & \multicolumn{2}{|c|}{ Average Reading Time/Page } & \multirow{2}{*}{$\begin{array}{c}\text { Number } \\
\text { Correct } \\
\text { on Final Test }\end{array}$} \\
\hline & $\begin{array}{c}\text { For First } 12 \\
\text { Pages of Text }\end{array}$ & $\begin{array}{l}\text { For Second } 12 \\
\text { Pages of Text }\end{array}$ & $\begin{array}{l}\text { For Second } 12 \\
\text { Pages of Text }\end{array}$ & $\begin{array}{c}\text { Over } 24 \text { Pages } \\
\text { of Text }\end{array}$ & \\
\hline Question After & 38 & 41 & 80 & 74 & \\
\hline Irrelevant Question & 36 & 35 & 72 & 70 & \\
\hline No Question With Probes & 38 & 37 & 65 & 69 & 2.11 \\
\hline No Question Without Probes & & & 67 & 69 & 2.88 \\
\hline
\end{tabular}


condition. Reaction times for the first 12 pages of probed text were compared with those for the second 12 pages. In the question after condition, the increase was reliable $\left[t(197)=2.39, \sigma_{\text {est }}=1.17\right]$. In the other groups, there was no reliable effect.

An unexpected effect observed in Experiment 1 was that reaction time in a questioned group increased markedly on the pages immediately following questions. This trend may be seen in Figure 1. On the next two pages of each three-page segment, a decline was evident but reaction time was still greater than in the group that was not questioned. The trend was not reliable in Experiment 1 when tested as the Groups by Pages interaction, but these effects were replicated in this experiment, as shown in Table 2. After Experiment 1 had been completed, it was discovered that McGaw and Grotelueschen (1973) had found the same effect in an experiment identical to this one except that learning was measured instead of cognitive load: Material from pages immediately following questions was more likely to be learned than material from other pages. The $\mathrm{McGaw}$ and Grotelueschen effects for learning were the same as the cognitive load results in this experiment, suggesting that cognitive load and learning are closely associated. These impressions based on Table 2 were confirmed by $t$ tests showing that in the question after condition reaction times were longer on the pages immediately following questions than (1) on the second page after questions $\left[\mathrm{t}(17)=3.91, \sigma_{\mathrm{est}}=1.38\right]$, or (2) on the third page after questions $[t(17)=3.14$, $\left.\sigma_{\text {est }}=1.43\right]$. In the no question with probes condition, reaction times on the three types of pages did not differ reliably, indicating that the nature of the material on the pages was not responsible for the effect. Immediately after questions, both cognitive capacity usage and learning (McGaw \& Grotelueschen, 1973) increased appreciably.

Table 1 shows reading time for each group. During the pages when the groups were treated differently, reading time in the question after group reliably exceeded that in the no question with probes group $\left[\mathrm{t}(34)=2.16, \sigma_{\text {est }}=6.94\right]$. The difference between the irrelevant question group and the no question with probes group was not reliable. Reading rates in the various groups ranged from 212 to 253 words $/ \mathrm{min}$. These are consistent with normal reading rates for college students (Coke, 1974). About 38\% of the inserted questions were answered correctly. Since these were short answer questions that could not be answered by guessing, this indicates that subjects were attending to the text.

Comparisons relevant to the effect of the secondary task on normal reading processing involved the no question with probes and no question without probes groups. Average reading times of these groups were identical, as shown in Table 1, indicating that the secondary task did not influence reading time. This
Table 2

Average Reaction Times on Pages Following Questions for Question After Condition and Counterpart Pages for No Question With Probes Condition

\begin{tabular}{lccc}
\hline Condition & $\begin{array}{c}\text { Page Imme- Second } \\
\text { diately } \\
\text { Following }\end{array}$ & $\begin{array}{c}\text { Third } \\
\text { After } \\
\text { Questions Questions }\end{array}$ & $\begin{array}{c}\text { Page } \\
\text { After }\end{array}$ \\
\hline Questions \\
\hline Question After & 44 & 39 & 40 \\
No Question With Probes & 38 & 37 & 37 \\
\hline
\end{tabular}

raises an important issue: The limited capacity hypothesis implies that when clicks are monitored, reading performance should suffer. But reading rate was evidently not slowed. This means that either the limited capacity hypothesis is wrong, or that there were too few clicks to cause appreciable interference with the reading rate aspect of reading performance. These alternative interpretations cannot be examined with the data from this study. The authors believe that it is possible to demonstrate an effect of click detection on reading speed if the clicks occur every few seconds. In these studies clicks occurred about every $34 \mathrm{sec}$ It can be concluded that infrequent clicks do not appreciably interfere with the reading task. The average reading time per page reported for these materials by Rothkopf (1966) for a reading-only control group corresponding to the no question without probes group was $70 \mathrm{sec}$, which corresponds very closely to the $69 \mathrm{sec}$ observed here.

Results of the comparison of final test scores did not provide a clear outcome. For the no question with probes condition, an average of 2.11 of the questions were correct on the final test, while for the no question without probes condition, 2.88 were correct. A $t$ test showed these did not differ $\left[\mathrm{t}(34)=1.57, \sigma_{\text {est }}=.49\right.$, $.10<\mathrm{p}<.25$ ]. However, a power analysis showed that the power of this test was only .16 , which is not large enough to provide much confidence in accepting the null hypothesis in this case. The effect of the secondary task on this second aspect of normal reading performance must therefore be regarded as an open question. It should be noted that, based on the observations of the authors, it appears very likely that if the frequency of probes were reduced below a certain level, the effect of adjunct questions on processing and probe reaction time would remain, and any possible effects of the probe on normal reading processing would be reduced to the vanishing point. The appropriate design would involve a parametric manipulation of probe frequency in pairs of question and no question groups, with a no question without probes group as control.

Additional replications. Since the time these experiments were completed, it has been standard practice in our laboratory in most of our experiments to present at the beginning of each experimental session 
Table 3

Average Reaction Times to Secondary Task for Passages Appearing Before and After Questions in Four Studies

\begin{tabular}{ccccc}
\hline & $\begin{array}{c}\text { Before } \\
\text { Study* }\end{array}$ & \multicolumn{2}{c}{ After Questions } & Percent- \\
\cline { 3 - 4 } & Questions & Immediately & Longer & age** \\
\hline 1 & 384 & 515 & 389 & 71 \\
2 & 286 & 511 & 389 & 79 \\
3 & 417 & 516 & 411 & 79 \\
4 & 398 & 517 & 381 & 83 \\
\hline
\end{tabular}

$* N=24$ in all studies. **Percentage of subjects with $R T$ for the passages immediately after questions higher than for either neighboring passage.

a short passage with probes followed by several difficult questions, before proceeding with the rest of the experiment. The purpose of this procedure is to give subjects practice with the reaction time task, to induce them to take the experiment seriously, to establish test expectations, to provide a common measurement of subjects in all groups, and to measure reading time to aid in the placement of probes in the remainder of the experiment. Immediately following the questions, more passages are presented. The reaction time data for the passages before the questions, immediately after the questions, and later passages can then be used to provide data on the generality of the tendency for reaction times to increase after inserted questions. Table 3 shows the results of four studies. In all cases a three-page segment of the marine biology passage was used as the initial passage. Average reaction times from the probe on the third page are shown. Then six short answer questions on the marine biology passage were asked. The next reaction times are from the passage immediately after the questions, and the next are from the passage after that. The latter two passages were all 150-word passages of different levels of difficulty. They changed from experiment to experiment. The effect was quite reliable. In lieu of significance tests, the percentage of subjects for whom the passages immediately after the questions had higher reaction times than either of the neighboring passages is posted.

Early in this research program, we followed the procedure of beginning the experimental passages immediately after the questions. The puzzlement caused by our peculiar results was dispelled when we realized that reaction times for the first passages after the questions were elevated. We have since followed the procedure of inserting nonexperimental filler passages in this position in order to allow dissipation of the transient effects of the inserted questions on reaction time.

\section{GENERAL DISCUSSION}

Adjunct questions caused increases in reaction time to the secondary task. These increases presumably reflect an increase in the amount of cognitive capacity used for reading.
This result is consistent with the hypothesis that learning from text is facilitated as more cognitive capacity is devoted to reading. One major hypothesis for the facilitative effects of adjunct questions on learning is that questioned readers "begin to process the text more thoroughly in order to be able to answer succeeding questions" (Anderson \& Biddle, 1975, p. 102). "More thoroughly" is the important part of this hypothesis.

Two aspects of thoroughness can be distinguished: the duration of cognitive processing and its type. With respect to the effects of adjunct question treatments on the duration of cognitive processing, the reading time observations in this experiment add only a small increment of data to what is already a formidable mass, complete with its own controversy about whether or not reading times increase in adjunct question treatments and whether the increase, if any, is a cause or a result of changes in processing or learning (Anderson \& Biddle, 1975; Carver, 1972; Rothkopf, 1974); a resolution of this will not be attempted here.

In the area of the type of cognitive activities induced by adjunct questions, this experiment provides evidence that cognitive capacity is used by the cognitive activities of a particular stage of reading processing. Before describing this evidence, the stages of reading processing will be delineated. Several cognitive activities are involved in reading. Each element of the printed page goes through three different cognitive processing stages before it can become part of the subject's store of knowledge. The stages of cognitive processing occur in a fixed order. First is the perceptual stage, during which the printed letters are recognized. Second is the literal comprehension stage, during which the groups of letters are looked up in the internal lexicon and the rules of grammar are applied to the strings of words, resulting in the comprehension of the plain sense of the sentences. Third is the elaborative stage, during which many different types of cognitive activities can occur, depending on the purpose of the reader. As an illustration of the elaborative stage processing, if the text is a story and the reader's purpose is entertainment, elaborative activities may involve constructing a mental image of the events in the story. To illustrate the serial aspect of the three processing stages, the elaborative stage process of imaging can only occur after the perceptual stage has been completed for each letter in that part of the text and after the literal comprehension stage has been completed for the sentences comprising the event in the story. That is, the image can only be created after the letters have been perceived, recognized, put into words and sentences, and the meaning of the sentences has been understood.

If the reader's purpose is different, different elaborative activities will occur. In the present studies, the reader's purpose was to learn the text, and elaborative processes in the adjunct question conditions probably involved such cognitive activities as deciding 
whether a sentence contained the type of information which had earlier appeared, and therefore might later appear, in an adjunct question, and processing it further if it did, and terminating processing if it did not (Rothkopf \& Bibiscos, 1967). The further processing given to test-relevant sentences may have included rereading them, developing mnemonic devices to recall test-relevant dates, names, and terms, creating images of scenes, relating sentences to earlier material in the text or to other knowledge about the topic, and judging if items of information had been memorized sufficiently to guarantee their reproduction on the anticipated test.

A certain amount is already known about the relation of cognitive capacity to the perceptual, literal comprehension, and elaborative stages of reading, and some additional information can be deduced from the present study. First, previous studies indicate that the perceptual (letter-encoding) stage of processing does not use cognitive capacity in the skilled reader. Of the many cognitive tasks that have been investigated with the secondary task technique (reviewed by Kahnemann, 1973; Kerr, 1973), only two have been found to require no cognitive capacity at all, and one of those is encoding letters (Posner \& Boies, 1971; Posner \& Klein, 1973).

The cognitive processing involved in the literal comprehension stage of reading, during which word meanings are looked up and grammar is applied to the word strings to get the plain sense of the clause or sentence, probably did not differ among the different groups in the present experiments, because the text itself, the main determiner of these processes, was identical in all conditions. Differences among the groups in cognitive capacity usage cannot be attributed to changes in the literal comprehension stage of processing. Consequently, no conclusions can be drawn about the cognitive capacity usage of the literal comprehension stage from these experiments.

Having eliminated the perceptual and literal comprehension stages as sources of the differences in cognitive capacity usage between adjunct question and other groups, the elaborative stage remains. Thus the present experiments demonstrate that cognitive capacity is used in the elaborative stage of reading.

Earlier in this discussion, several cognitive activities were identified which may be involved in the elaborative processing that occurs after adjunct questions have been presented. These candidate cognitive activities were identified by the first author, partly by reference to the effect of independent variables in adjunct question experiments, partly by interviewing subjects exposed to adjunct questions, and partly by reasoning about the prerequisites to activities identified in the other two ways. These may not constitute a correct or complete list of the cognitive activities induced by adjunct questions, but they are sufficient for illustrating the several ways in which cognitive capacity might be involved in learning. First, it is conceivable that cognitive capacity could be used only in the activities that are preparatory to learning, such as identifying test-relevant sentences, and not in activities that are directly involved in memory storage and learning, such as memorizing facts and dates. Alternatively, cognitive capacity could be used only in the activities that cause memory storage, or it could be used in both types of activities. Further research will be directed toward isolating cognitive activities and determining which cognitive activities cause learning and which use cognitive capacity.

Finally, in introducing a new measurement technique, it is appropriate to consider in detail the assumptions relating the measured quantity to the phenomena of interest. The secondary task technique used to measure cognitive capacity usage in this study involves several assumptions. One is that the central processing system has limited capacity. This assumption is widely accepted because the alternative hypothesis of an unlimited capacity is implausible. A second assumption is that the primary and secondary tasks require the same central processing system. The task of reading prose and that of responding to clicks are so different that it is difficult to conceive specifically of any definite mechanism that could be required by both of them. But it is a matter of common observation that tasks which seem at first sight to be widely different turn out quite often to interfere with each other if either of them requires any capacity at all, as can easily be shown by asking someone who is walking to do a problem in mental arithmetic. Almost always they will stop walking, indicating that even a task as well established as walking uses mental capacity.

Moreover, it has been shown experimentally that click probes of the type used in this study show all the signs of using the same limited-capacity central processor as a variety of primary tasks (Kahneman, 1973; Kerr, 1973). This finding does not reduce the difficulty of conceiving of a mechanism that is required for both tasks. The nature of this mechanism is, of course, a central problem of cognitive psychology. A third assumption is that, as more capacity is devoted to the primary task, less is available for secondary task performance. This follows from the preceding assumptions, if it is assumed that capacity is fixed.

Finally, it is assumed that the decrements in reaction time reflect reductions in the mental capacity that is available for the secondary task. In this connection, Kahneman (1973) has distinguished between capacity interference and structural interference. Structural interference refers to decrements in secondary task performance that occur because the two tasks use the same specific perceptual, memory, or response systems.

If, for example, both the primary and secondary tasks require use of the eye for perceiving, or of the same finger for responding, structural interference is implicated in decrements in secondary task performance. 
In examining the issue of whether structural interference was involved in the decrements in secondary task performance observed in this study, the primary question is whether the two tasks used the same perceptual, memory, or response systems. The tasks utilized different sensory modalities, the eyes and the ears, so structural interference at the perceptual level seems unlikely. The memory system is probably importantly involved in reading. The secondary task appears to use the memory system to the minimum extent possible, short of not using it at all. The response system involved in reading is primarily covert, except for eye movements and subvocalizing, which use effector units that are quite distinct from those involved in keypressing. These considerations do not lend support to the hypothesis that structural interference is importantly involved in the reaction time decrements observed in the secondary task used here. In any case, since all groups had the same secondary task, structural interference was presumably equated for the groups, so that any remaining differences were due to other sources. In summary, the assumptions that provide the rationale for using the secondary task technique to determine the cognitive capacity utilized in the primary task appear to be justified in the present study.

\section{REFERENCES}

Anderson, R. C.. \& Biddle, W. B. On asking people questions about what they are reading. In G. Bower (Ed.),
Psuchology of learning and motivation (Vol. 9). New York: Academic Press. 1975.

CARson. R. L. The sea around us. New York: Oxford University Press. 1951.

CARver. R. P. A critical review of mathemagenic behaviors and the effect of questions upon the retention of prose materials. Journal of Reading Behavior. 1972, 4. 93-119.

COKE. E. V. The effects of readability on oral and silent reading rates. Journal of Educational Psychology. 1974. 66. 406.409 .

Kahneman, D. Attention and effort. Englewood Cliffs, N.J: Prentice.Hall, 1973

KERR. B. Processing demands during mental operations. Memon \& Cognition. 1973. 1. 401-412.

McGaw. B., \& Grotelueschen. A. Direction of the effect of questions in prose material. Journal of Educational Psychology, 1973, 63. 580-588.

Posner. M. I.. \& Boles, S. J. Components of attention. Psychological Review, 1971, 78, 391-408.

PosNer, M. I., \& KLEIN, R. On the functions of conscious ness. In S. Kornblum (Ed.), Attention and performance IV. New York: Academic Press. 1973.

Roтнкорғ. E. Z. Learning of written instructive materials: An exploration of the control of inspection behavior by testlike events. American Educational Research Journal. 1966. 3. $241-249$.

Rothkopf, E. Z. Barbarism and mathemagenic activities: Comments on criticism by Carver. Joumal of Reading Behavior, 1974, 6, 1-8.

Rothxopf, E. Z., \& Bibiscos, E. E. Selective facilitative effects of interspersed questions on learning from written materials. Journal of Educational Psychology. 1967, 58. $36-61$.

(Received for publication August 2, 1977; accepted January 6, 1978.) 